\title{
Composite fermion model for entanglement spectrum of fractional quantum Hall states
}

\author{
Simon C. Davenport, ${ }^{1}$ Iván D. Rodríguez, ${ }^{2}$ J. K. Slingerland, ${ }^{3,4}$ and Steven H. Simon ${ }^{5}$ \\ ${ }^{1}$ T.C.M. Group, Cavendish Laboratory, J.J. Thomson Avenue, Cambridge CB3 OHE, United Kingdom \\ ${ }^{2}$ Max-Planck-Institut für Quantenoptik, 85748 Garching, Germany \\ ${ }^{3}$ Department of Mathematical Physics, National University of Ireland, Maynooth, Ireland \\ ${ }^{4}$ Dublin Institute for Advanced Studies, School of Theoretical Physics, 10 Burlington Road, Dublin, Ireland \\ ${ }^{5}$ Rudolf Peierls Centre for Theoretical Physics, 1 Keble Road, Oxford OX1 3NP, United Kingdom
}

(Received 24 June 2015; published 30 September 2015)

\begin{abstract}
We show that the entanglement spectrum associated with a certain class of strongly correlated many-body states - the wave functions proposed by Laughlin and Jain to describe the fractional quantum Hall effect-can be very well described in terms of a simple model of noninteracting (or weakly interacting) composite fermions.

DOI: 10.1103/PhysRevB.92.115155

PACS number(s): 73.43.-f, 03.67.Mn, 05.30.Pr
\end{abstract}

Understanding of strongly correlated many-body quantum states has been significantly enhanced in recent years by the introduction of the entanglement spectrum (ES), i.e., the spectrum of eigenvalues of the reduced density matrix of a subsystem [1]. The scrutiny of the ES has been particularly fruitful in the study of fractional quantum Hall (FQH) wave functions-the archetype for topological phases of matter resulting from strong interaction effects-where this analysis often reveals a special underlying entanglement structure useful for identifying and classifying different topological phases [1-6].

For FQH states in particular it has been established that there is a deep connection between the entanglement structure of bulk wave functions (as seen in their entanglement spectra [7-9]) and the structure of edge excitations [10-13]. Central to this connection so far have been arguments based on the common conformal field theory (CFT) description of the bulk and edge physics, which is known to apply for the class of wave functions that can be constructed in terms of expansion functions know as "conformal blocks". The ES for this class of what we refer to as "simple" FQH states has been extensively studied using CFT methods [4,5,9].

Largely separate from these developments, it has been well documented that the composite fermion model of the FQH effect can successfully account for many experimentally observed features of the effect, particularly in the lowest Landau level (LL) $[14,15]$. It does so by positing that they are due to the integer quantum Hall (IQH) effect of noninteracting (or weakly interacting) quasiparticles known as composite fermions. Although composite fermion theory presents an appealingly simple physical picture to explain the FQH effect, it has proved to be very challenging to relate the many-body composite fermion wave functions (which we refer to as Jain states $[14,15])$ to constructs in CFT. Although such constructs do exist [16-19], they are somewhat complicated, and this has so far limited the effectiveness of CFT machinery in describing the ES of the Jain states. In this sense the Jain states are generally not simple FQH states.

In this paper we present a very different approach to constructing entanglement spectra that bypasses the potential difficulty associated with explicitly writing wave functions in terms of conformal blocks. We show that the low-lying (highest weight) part of the ES of the Jain FQH states can be accurately described by a modified ES of noninteracting (or weakly interacting) composite fermions in filled LLs (in other words a modified ES of the IQH states [20,21]). To demonstrate the effectiveness of our method, we focus on presenting results for two fundamental examples, namely, the Laughlin state at filling factor $v=1 / 2$ for bosons [22] (a simple FQH state that was previously studied using CFT methods [9]) and the Jain state at $v=2 / 3$ for bosons (a nonsimple FQH state). (The method applies equally well to fermionic FQH states.) Our results for the Laughlin case are in good agreement with the approach based on CFT, but the real advantage of our method becomes evident for the Jain states where the CFT approach appears much more complicated and has therefore not been worked out.

In the spirit of the original composite fermion model [14,15], we start with the ES for the IQH states [20,21]. We briefly review its derivation here. For simplicity we consider spinless particles. Also, to remove additional complications due to edge physics we consider a system without physical edges. A standard technique to achieve this is to solve the problem on the surface of a sphere [23]. Here we have an integer $n$ LL problem (where the filling factor is $v=n$ ) for $N$ particles on a sphere of radius $\sqrt{Q}$ (in units of magnetic length) that encloses a fictitious magnetic monopole of strength $2 Q=\left(N-n^{2}\right) / n$. There is rotational symmetry about the $z$ axis, which leads to the single-particle orbitals of the problem being labeled by the $z$ component of angular momentum $m=$ $-(2 Q+\sigma) / 2,-(2 Q+\sigma) / 2+1, \ldots,(2 Q+\sigma) / 2$ in addition to a LL index $\sigma=0,1, \ldots, n-1$. The single-particle orbitals are $\phi_{m, \sigma}(\mathbf{r})$, where $\mathbf{r}$ lies on the surface of the sphere [technically, $\phi_{m, \sigma}(\mathbf{r})$ are monopole harmonics [24]. The monopole harmonics also depend explicitly on $Q$, but for simplicity we suppress this dependence].

To connect with existing calculations, we focus on the entanglement spectrum for wave functions in real space (as opposed to, e.g., momentum space), which leads to the real-space entanglement spectrum (RSES) [4-6].

We consider cuts along lines of latitude so that that rotational invariance about the $z$ axis is preserved and the $z$ component of angular momentum $L_{z}$ remains a good quantum number. Once the system is cut, $L_{z}$ is bipartitioned as $L_{z}=L_{z}^{A}+L_{z}^{B}$. Additionally, the total particle number is bipartitioned as $N=N_{A}+N_{B}$.

For the full fermionic $N$-particle system, the basis for the Hilbert space consists of Slater determinants built from the orbitals $\phi_{m, \sigma}(\mathbf{r})$. For the $A$ and $B$ subsystems, the 
restrictions of these orbitals to the regions $A$ and $B$ still form a complete single-particle basis, although the restricted orbitals must be renormalized to account for only part of the single-particle weight being in each subsystem. Once restricted to a subsystem, the orbitals $\phi_{m_{1}, \sigma_{1}}$ and $\phi_{m_{2}, \sigma_{2}}$ are orthogonal whenever $m_{1} \neq m_{2}$ (because the cut is made to preserve $L_{z}$ ) but are generally not orthogonal whenever $\sigma_{1} \neq \sigma_{2}$ (although they remain linearly independent in this case). The Fock space of the $A$ and $B$ subspaces is spanned by Slater determinants in terms of these restricted orbitals.

We can now write the Schmidt decomposition of state $|\psi\rangle$ as

$$
|\psi\rangle=\sum_{i} e^{-\xi_{i} / 2}\left|\psi_{i}^{A}\right\rangle \otimes\left|\psi_{i}^{B}\right\rangle
$$

where $\left|\psi_{i}^{A}\right\rangle$ and $\left|\psi_{i}^{B}\right\rangle$ belong to the Fock spaces for the $A$ and $B$ subsystems that we have just described. The nonzero Schmidt coefficients $e^{-\xi_{i} / 2}$ are conveniently written in terms of the entanglement energies $\xi_{i}$. The index $i$ labels the vectors in the Schmidt basis for the $A$ system (at least those with nonzero Schmidt coefficients). Since $N_{A}$ and $L_{z}^{A}$ are conserved by the cut, we can choose the Schmidt basis to consist of eigenstates of $N_{A}$ and $L_{z}^{A}$. We can then write $\xi_{N_{A}, L_{z}^{A}, i}$ for the entanglement energy of the $i$ th Schmidt state with given $N_{A}$ and $L_{z}^{A}$. In fact, with any Schmidt state with $N_{A}$ particles and given $L_{z}^{A}$, we can associate an $N_{A}$ tuple of single-particle momenta $\mathbf{m}=\left(m_{1}, \ldots, m_{N_{A}}\right)$ with $L_{z}^{A}=$ $\sum_{p} m_{p}$ and $m_{1}<m_{2}<\cdots<m_{N_{A}}$. If there is only a single LL involved, the Schmidt state is just the unique Slater determinant in the Fock space of system $A$ labeled by $\mathbf{m}$, and we can replace the label $i$ above by $\mathbf{m}$. With multiple LLs, there will be a number of Schmidt states associated with each $\mathbf{m}$ : These are superpositions of the Slater determinants with this $\mathbf{m}$ and different choices of the numbers of particles in each LL.

For a noninteracting system, one can find exact expressions for the entanglement energies and Schmidt states using an argument proposed by Peschel [25]. If one constructs the correlation matrix,

$$
C_{m_{1}, \sigma_{1}, m_{2}, \sigma_{2}}=\delta_{m_{1}, m_{2}} \int_{A} \phi_{m_{1}, \sigma_{1}}^{*}(\mathbf{r}) \phi_{m_{1}, \sigma_{2}}(\mathbf{r}) d \mathbf{r},
$$

then the eigenvalues $\lambda_{m, \sigma}$ of $C$ are related to the entanglement energies by

$$
\xi_{i}=\sum_{m, \sigma} o_{m, \sigma, i} \epsilon_{m, \sigma}+\text { constant }
$$

where the single-particle entanglement energy function $\epsilon_{m, \sigma}$ is defined by

$$
\epsilon_{m, \sigma}=\log \left[\frac{1-\lambda_{m, \sigma}}{\lambda_{m, \sigma}}\right] .
$$

Note that now $\sigma$ labels the eigenstates of the correlation matrix at given $m$ rather than a LL. The full Schmidt states are Slater determinants built from these eigenstates, and $o_{m, \sigma, i}$ denotes the occupation number of the correlation matrix eigenstates labeled by $(m, \sigma)$ in the Schmidt state with label $i$. Examples of the RSES for the $v=1$ and $v=2$ IQH states calculated using this method are included in Figs. 1(a) and 2(a) for later comparison with our results for the FQH case.
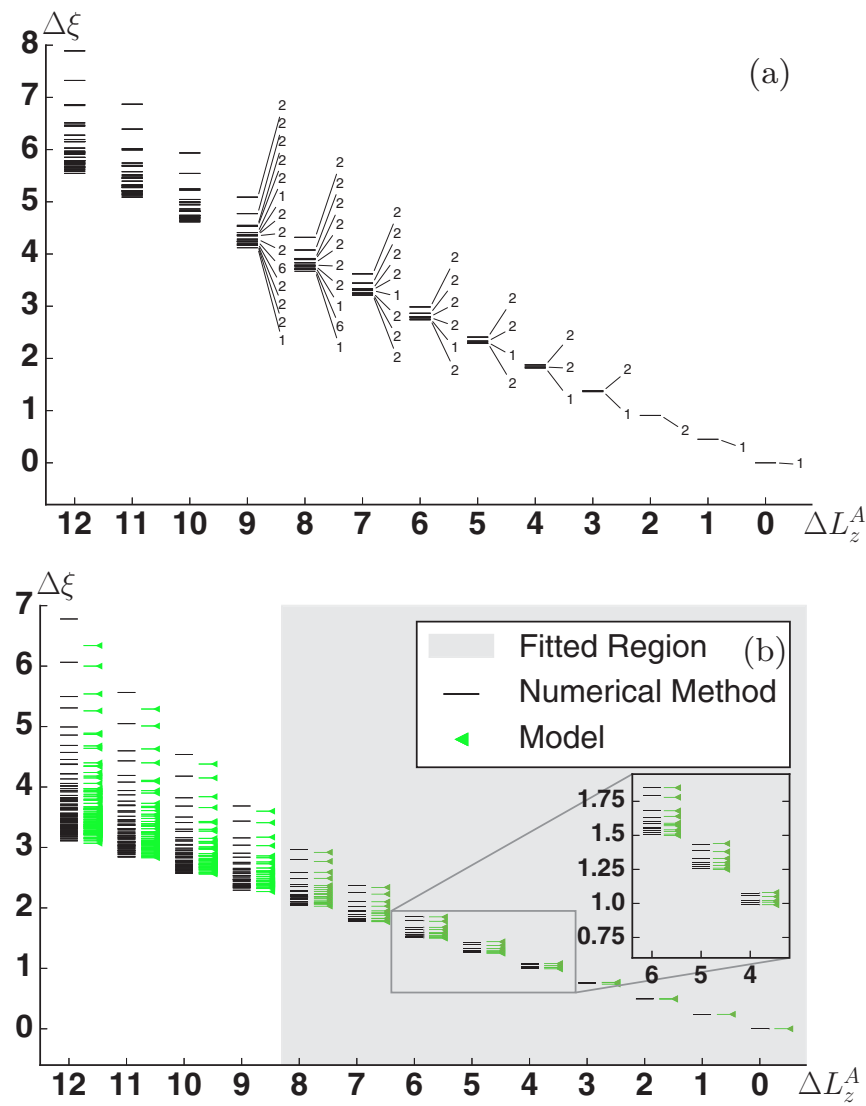

FIG. 1. (Color online) (a) Exact RSES for the $v=1$ state for $N=50$ particles with equal-size $A$ and $B$ regions and $N_{A}=25$. Embedded number labels indicate the degeneracy of $\xi$ (up to $\Delta L_{z}^{A}=8$ ) [26]. (b) Fit of the single-particle entanglement energy model [Eq. (5) in terms of the composite fermion angular momentum $m^{\prime}$ and truncated at order $m^{\prime 3}$ ] to the ES of the $v=1 / 2$ Laughlin state calculated numerically for the same cut and system size using the method in Ref. [27]. Fitting parameter data are included in Appendix B. Entanglement energies $\xi$ and angular momentum labels $L_{z}^{A}$ are relative to the lowest-lying state $\xi_{0}$ in the lowest angular momentum sector $L_{0}^{A}$, i.e., $\Delta \xi=\xi-\xi_{0}$ and $\Delta L_{z}^{A}=L_{0}^{A}-L_{z}^{A}$.

We now come to the statement of our main result: For the class of FQH states proposed by Laughlin and Jain to describe the FQH effect in the lowest LL at filling factors $v=$ $n^{\prime} /\left(2 n^{\prime}+1\right)$ [for fermions] and $v=n^{\prime} /\left(n^{\prime}+1\right)$ [for bosons] with $n^{\prime}$ as an integer, the low-lying $\xi$ in the associated RSES can be accurately described by the same model as the IQH states [Eq. (3)] but a different single-particle entanglement energy function [Eq. (4)]. In addition, when multiple effective LLs are present in the Jain wave functions, one also needs to take into account a simple exchange-entanglement-energy term that we will shortly describe with an example.

In this connection the LL index $\sigma$ for the IQH wave functions becomes the effective LL index of the Jain states $\sigma^{\prime}=0,1, \ldots, n^{\prime}-1$, the magnetic field described by the monopole strength $Q$ is replaced with an effective magnetic field and an effective monopole strength $Q^{\prime}=\left(N-n^{\prime 2}\right) / n^{\prime}$, and the effective single-particle eigenstates are labeled by $m^{\prime}=$ $-\left(2 Q^{\prime}+\sigma^{\prime}\right) / 2,-\left(2 Q^{\prime}+\sigma^{\prime}\right) / 2+1, \ldots,\left(2 Q^{\prime}+\sigma^{\prime}\right) / 2$.

It is useful to expand $\epsilon_{m, \sigma}$ from Eq. (4) as a power series in $m$ about the midpoint $m=0$ as it turns out that we need to 

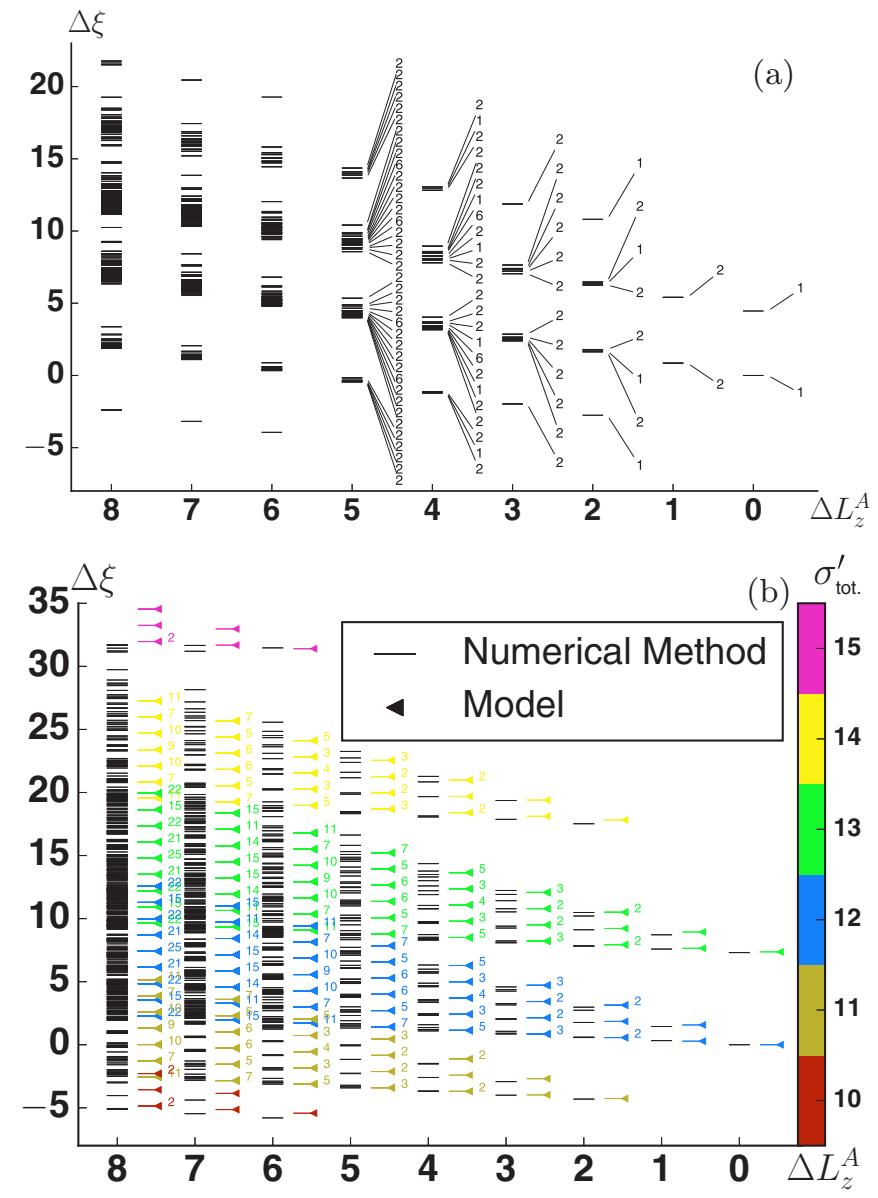

FIG. 2. (Color online) (a) Exact RSES for the $v=2$ state for $N=48$ particles with equal-size $A$ and $B$ regions and $N_{A}=24$. (b) Fit of the single-particle entanglement energy model [Eq. (8)] to the $v=2 / 3$ Jain state calculated numerically for the same cut and system size using the method in Ref. [27]. The fitting parameter data are included in Appendix B. $\sigma_{\text {tot. }}^{\prime}$ labels the number of particles in the upper effective LL for each $\xi$. Entanglement energies $\xi$ and angular momentum labels $L_{z}^{A}$ are relative to the lowest-lying state $\xi_{0}$ in the lowest angular momentum sector $L_{0}^{A}$, i.e., $\Delta \xi=\xi-\xi_{0}$ and $\Delta L_{z}^{A}=L_{0}^{A}-L_{z}^{A}$. Number labels embedded in the plots indicate the degeneracy of $\xi$ where ambiguous [26].

keep only very few terms in this expansion for a very accurate description of $\epsilon_{m, \sigma}$. For example, in the case of a single LL ( $v=n=1$ for spinless particles) or a single effective $\operatorname{LL}\left(n^{\prime}=\right.$ 1 and replacing $m$ with $m^{\prime}$ in the expansion),

$$
\epsilon_{m, \sigma} \approx a_{0}+a_{1} m+a_{2} m^{2}+a_{3} m^{3}+\cdots,
$$

where, for the IQH case, $a_{0}, a_{1}$, etc., are functions that can be expressed in terms of the eigenvalues of the matrix $C$ and its derivatives (they also depend on the system size via their dependence on $Q$ ). For two filled LLs ( $v=n=2$ for spinless particles) or two effective LLs $\left(n^{\prime}=2\right)$ the expansion becomes instead

$$
\begin{aligned}
\epsilon_{m, \sigma} \approx & a_{0,0}+a_{0,1} m+a_{0,2} m^{2}+a_{0,3} m^{3}+\cdots \\
& +[\sigma-1 / 2]\left\{a_{1,0}+a_{1,1} m+a_{1,2} m^{2}+\cdots\right\} .
\end{aligned}
$$

In our approach for the FQH case the coefficients $a_{j, k}$ now play the role of fitting parameters in a model given by truncating the single-particle energy function at a low degree in $\mathrm{m}^{\prime}$. Their values are determined by fitting this model to the RSES generated numerically from a microscopic construction of the FQH state. Further details of the fitting procedure are given in Appendix A. To obtain a RSES to fit to for large system sizes we use the method proposed in Ref. [27] (which works most efficiently for bosonic systems).

To illustrate how our approach works we present two examples of applications to bosonic FQH states where we choose symmetrical equal-sized regions $A$ and $B$ (i.e., a cut along the equator of the sphere) and $N_{A}=N_{B}=N / 2$ [26]. The method also applies for more general cuts.

First we consider the RSES of the Laughlin state of bosons at filling factor $v=1 / 2$ (equivalently the $n^{\prime}=1$ Jain state). The RSES for $v=1 / 2$ is accurately described by the single-particle energy function in Eq. (5), written in terms of the composite fermion angular momentum labels $m^{\prime}$ and truncating the expansion at order $m^{\prime 3}$. In this truncated expansion, the coefficients $a_{1}, a_{2}$, and $a_{3}$ are free fitting parameters ( $a_{0}$ is fixed by normalization: See Appendix A). An example fit to the numerically calculated RSES of the $v=1 / 2$ state is shown in Fig. 1(b). The fitting parameter data are included in Appendix B. We find that by far the most dominant contribution comes from the $a_{1}$ term, indicating that the spectrum is almost linear. In Fig. 1(a) we show the RSES of the $v=1 \mathrm{IQH}$ state for comparison.

The Laughlin state falls into the class of simple FQH states, and its RSES has been studied previously using CFT techniques. In particular Ref. [9] describes a method to model the Laughlin RSES in terms of the eigenstates of an entanglement Hamiltonian given by writing down every allowable CFT operator (in this case chiral boson operators, their derivatives, and powers) order by order in their scaling dimension (with terms at lower scaling dimension providing more relevant contributions). These operators come with unknown coefficients that can be fitted to the numerically calculated RSES using a similar procedure to that described in Appendix A. In Ref. [9] it was shown that the RSES of the $v=1 / 2$ state can be very accurately described by including allowable CFT operators up to scaling dimension 2, leading to an entanglement Hamiltonian containing three terms (and therefore three fitting parameters). We have checked that for the same data set as used here [Fig. 1(b)] the fit of this truncated entanglement Hamiltonian to the RSES is in excellent agreement with our noninteracting model. One could in principle reproduce our model directly (allowing for additional interaction terms) by fermionizing Dubail-ReadRezayi's entanglement Hamiltonian [9]. The fact that our result agrees closely with the numerically calculated RSES implies that any additional interaction terms must only be small corrections, which justifies the assumptions made by the composite fermion approach.

Our second example is the RSES of the $n^{\prime}=2$ Jain state of bosons at filling factor $v=2 / 3$. Our technique was not able to improve upon CFT in treating simple FQH wave functions, but here it excels. We now use the single-particle energy from the $v=2 \mathrm{IQH}$ state [Eq. (6)], but we augment it to take into account an exchangelike interaction (treated at the level of "mean-field theory") for fermions in different LLs. This term can alternatively be thought of as a "charging 
energy". Our ansatz to describe the RSES of the $v=2 / 3$ Jain state is

$$
\xi_{N_{A}, L_{z}^{A}, i}=\sum_{m^{\prime}, \sigma^{\prime}} n_{m^{\prime}, \sigma^{\prime}} \epsilon_{m^{\prime}, \sigma^{\prime}}+c(\Delta N)^{2},
$$

with the single-particle energy function given by

$$
\epsilon_{m^{\prime}, \sigma^{\prime}} \approx a_{0,1} m^{\prime}+\left[\sigma^{\prime}-1 / 2\right]\left\{a_{1,0}+a_{1,1} m^{\prime}\right\},
$$

and the exchange-interaction term given by

$$
\Delta N=\sum_{m^{\prime}, \sigma^{\prime}} n_{m^{\prime}, \sigma^{\prime}}\left[\sigma^{\prime}-1 / 2\right] .
$$

This ansatz requires four fitting parameters $a_{0,1}, a_{1,0}, a_{1,1}$, and $c$ (the $a_{0,0}$ parameter can be fixed by normalization).

In Fig. 2(b) we show an example fit to the RSES of the $2 / 3$ state for a large system of $N=48$ particles. The fitting parameter data are included in Appendix B. We observe that the most dominant contribution arises from the coefficient $a_{1,0}$, which can be thought of as an entanglement "cyclotron energy" term. We also find that the coefficient of the exchange interaction $c$ is positive (so due to this additional term, the branches in the RSES for $v=2 / 3$ are further apart than they would have been otherwise). Note that there are degeneracies in the fitted entanglement energy eigenvalues, but these can be lifted by truncating the entanglement energy expansion at higher order in $m^{\prime}$. For comparison, in Fig. 2(a) we plot the RSES of the $v=2 \mathrm{IQH}$ state.

To summarize, we have described how a RSES of noninteracting composite fermions can be constructed to accurately approximate the RSES for certain strongly correlated FQH states. Key to this construction is the observation that the single-particle entanglement energy function underlying the description of the RSES for the IQH states can be simply modified in order to describe the RSES of FQH states treating the many-body RSES within a noninteracting approximation and allowing for very basic mean-field-theory-like exchange energy corrections in the multi-LL case. The quality of this approximation could be improved by allowing for higher-order corrections to the underlying entanglement energy function (at the cost of needing more fitting parameters).

We note that this description of the RSES closely parallels the description of the real energy spectrum of the edge of a Hall droplet in terms of composite fermions (see, e.g., Ref. [28]).

We thank S. Sondhi for helpful discussions. S.C.D. was supported by EPSRC Grant No. EP/J017639/1. I.D.R. was supported by EU Integrated Project SIQS. J.K.S. was supported by Science Foundation Ireland Principal Investigator Award No. 12/IA/1697. S.H.S. was supported by EPSRC Grants No. EP/I032487/1 and No. EP/I031014/1. We acknowledge use of the Hydra computer cluster at the Rudolf Peierls Centre for Theoretical Physics. Statement of compliance with EPSRC policy framework on research data: This paper reports theoretical work that does not require supporting research data.

\section{APPENDIX A: FITTING ALGORITHM}

In this appendix we will briefly describe our procedure for evaluating the goodness of fit of the RSES to the single-particle models proposed in this paper.
The problem is a many-parameter optimization where we aim to minimize a weighted sum of squared differences between corresponding $\xi$ in the lowest-lying part of the RSES and single-particle energy spectrum for a given set of fitting parameters $\left\{a_{j, k}\right\}$. By corresponding $\xi$ we mean that, for comparison, we normalize both the model spectrum and the numerically calculated spectrum such that the lowest lying $\xi$ in the lowest $\Delta L_{z}^{A}$ sector is set to zero. Then we order the set of $\xi$ in the model spectrum (call them $\xi_{\text {model }}$ ) and the lowest-lying part of the RSES (call them $\xi_{\text {RSES }}$ ) by their $\Delta L_{z}^{A}$ values, then for each sector we sort in order of increasing $\xi_{\text {RSES }}$ or $\xi_{\text {model }}$ value, and finally we take the sum of the squared difference between the lowest $\xi_{\text {RSES }}$ and the lowest $\xi_{\text {model }}$, the difference between the next lowest $\xi_{\text {RSES }}$ and the next lowest $\xi_{\text {model }}$, and so on. This sum can also be weighted in various ways, for instance, if we want to give increased importance to matching up states with the lowest values of $\xi$. In general, therefore, we aim to minimize a fitting function of the form

$$
R\left(\left\{a_{j, k}\right\}\right)=\sum_{i}\left[\xi_{\mathrm{RSES}}^{i}-\xi_{\text {model }}^{i}\left(\left\{a_{j, k}\right\}\right)\right]^{2} W_{i},
$$

where $\left\{a_{j, k}\right\}$ denotes the set of free parameters in the model and the index $i$ appearing in the sum denotes the $i$ th value of $\xi_{\text {model }}, \xi_{\text {RSES }}$ within the ordered set (ordered in the sense described above). The factor $W_{i}$ assigns an optional weight to each term in the sum. In addition, we only include entanglement spectrum eigenvalues below a certain specified cutoff sector $\Delta L_{\text {cutoff }}^{A}$, i.e., $W_{i}=0$ if it refers to a state with $\Delta L_{z}^{A}>\Delta L_{\text {cutoff }}^{A}$.

In our application we always include a factor in $W_{i}$ that divides out the number of states in each sector (i.e., for every $\xi_{\mathrm{EH}}^{i}$ labeled by the same $\Delta L_{z}^{A}$ we divide by a factor of $N_{\Delta L_{z}^{A}}$ that counts the total number of $\xi_{\text {model }}$ with that of $\Delta L_{z}^{A}$ ). The reason for doing this is because otherwise the fitting function would assign overwhelmingly more weight to fitting the higher sectors (because the counting of states in the RSES grows superpolynomially). Once the $N_{\Delta L_{z}^{A}}$ factor is divided out, each sector counts for the same total weight in Eq. (A1), i.e., $\sum_{i \text { with } \Delta L_{z}^{A}} W_{i}=1$ for all $\Delta L_{z}^{A}$. Another aspect to consider is that, in the RSES, linearly smaller $\xi$ correspond to exponentially greater coefficients in the Schmidt decomposition. In this sense, it should be more important physically to match up the lower lying $\xi$. For this reason, we might also consider including a factor $\exp \left(-\xi_{\mathrm{RSES}}^{i}\right)$ in our weight function. This addition works well for the Laughlin case, however the Jain case is more complicated due to the presence of the branch structure in the RSES. Consequently, for studying the Laughlin state we use the fitting function,

$$
R_{\text {Laughlin }}=\sum_{i \mid \Delta L_{z}^{A} \leqslant \Delta L_{\text {cutoff }}^{A}} \frac{\left(\xi_{\text {RSES }}^{i}-\xi_{\text {model }}^{i}\right)^{2} e^{-\xi_{\text {RSES }}^{i}}}{N_{\Delta L_{z}^{A}}},
$$

whereas for fitting the Jain state we find that for fitting all branches with equal weight it is better to use

$$
R_{\mathrm{Jain}}=\sum_{i \mid \Delta L_{z}^{A} \leqslant \Delta L_{\text {cutoff }}^{A}} \frac{\left(\xi_{\mathrm{RSES}}^{i}-\xi_{\text {model }}^{i}\right)^{2}}{N_{\Delta L_{z}^{A}}} .
$$


The quality of a given fitting model (e.g., a given set of fitting parameters $\left.\left\{a_{j, k}\right\}\right)$ can be assessed by the minimal value of the fitting function obtained for that model. The lower the minimal value of the fitting function, the better the quality of the fit.

In order to solve the minimization problem we use the Powell method provided by Scientific Python (SCIPY) version 0.11 and above, which involves a sequential one-dimensional minimization of each fitting parameter. The Powell method is found to be numerically stable for this problem. One also has to take steps to avoid finding the local rather than the global minimum, which we achieve by running the procedure a large number of times with different random starting parameters.

\section{APPENDIX B: FITTING PARAMETER DATA}

In this appendix we tabulate the values of the fitting parameters $a_{j, k}$ obtained in the single-particle energy fits shown in Figs. 1(b) and 2(b). We also include the corresponding values of the fitting function $R\left(\left\{a_{j, k}\right\}\right)$ defined in Appendix A to assess the relative quality of those fits.
Example fitting parameter values and $R_{\text {Laughlin }}$ [Eq. (A2)] values in the single-particle model proposed in this paper for the Laughlin $v=1 / 2$ state. Corresponding results are plotted in Fig. 1(b). Smaller values of $R_{\text {Laughlin }}$ indicate a better quality of fit:

\begin{tabular}{ccccc}
$a_{1}$ & $a_{2}$ & $a_{3}$ & $\Delta L_{\text {cutoff }}^{A}$ & $R_{\text {Laughlin }}$ \\
\hline 0.243 & 0.00130 & 0.00214 & 8 & $8.12 \times 10^{-5}$
\end{tabular}

Example fitting parameter values and $R_{\text {Jain }}$ [Eq. (A3)] values in the single-particle model proposed in this paper for the Jain $v=2 / 3$ state. Corresponding results are plotted in Fig. 2(b). Smaller values of $R_{\text {Jain }}$ indicate a better quality of fit:

\begin{tabular}{cccccc}
$a_{0,1}$ & $a_{1,0}$ & $a_{1,1}$ & $c$ & $\Delta L_{\text {cutoff }}^{A}$ & $R_{\text {Jain }}$ \\
\hline 0.9279 & 3.371 & 0.6429 & 0.1557 & 8 & 0.945
\end{tabular}

[1] H. Li and F. D. M. Haldane, Phys. Rev. Lett. 101, 010504 (2008).

[2] M. Haque, O. Zozulya, and K. Schoutens, Phys. Rev. Lett. 98, 060401 (2007).

[3] O. S. Zozulya, M. Haque, K. Schoutens, and E. H. Rezayi, Phys. Rev. B 76, 125310 (2007).

[4] J. Dubail, N. Read, and E. H. Rezayi, Phys. Rev. B 85, 115321 (2012).

[5] A. Sterdyniak, A. Chandran, N. Regnault, B. A. Bernevig, and P. Bonderson, Phys. Rev. B 85, 125308 (2012).

[6] I. D. Rodríguez, S. H. Simon, and J. K. Slingerland, Phys. Rev. Lett. 108, 256806 (2012).

[7] X.-L. Qi, H. Katsura, and A. W. W. Ludwig, Phys. Rev. Lett. 108, 196402 (2012).

[8] B. Swingle and T. Senthil, Phys. Rev. B 86, 045117 (2012).

[9] J. Dubail, N. Read, and E. H. Rezayi, Phys. Rev. B 86, 245310 (2012).

[10] A. Lopez and E. Fradkin, Phys. Rev. B 44, 5246 (1991).

[11] G. Moore and N. Read, Nucl. Phys. B 360, 362 (1991).

[12] X.-G. Wen, Int. J. Mod. Phys. B 06, 1711 (1992).

[13] A. Cappelli, C. A. Trugenberger, and G. R. Zemba, Nucl. Phys. B 396, 465 (1993).

[14] J. K. Jain, Composite Fermions (Cambridge University Press, Cambridge, U.K., 2007).

[15] J. K. Jain, Phys. Rev. Lett. 63, 199 (1989).
[16] T. H. Hansson, C.-C. Chang, J. K. Jain, and S. Viefers, Phys. Rev. Lett. 98, 076801 (2007).

[17] T. H. Hansson, C.-C. Chang, J. K. Jain, and S. Viefers, Phys. Rev. B 76, 075347 (2007).

[18] E. J. Bergholtz, T. H. Hansson, M. Hermanns, A. Karlhede, and S. Viefers, Phys. Rev. B 77, 165325 (2008).

[19] A. Cappelli, J. Phys. A: Math. Theor. 46, 012001 (2013).

[20] I. D. Rodríguez and G. Sierra, Phys. Rev. B 80, 153303 (2009).

[21] I. D. Rodríguez and G. Sierra, J. Stat. Mech.: Theory Exp. (2010) P12033.

[22] R. B. Laughlin, Phys. Rev. Lett. 50, 1395 (1983).

[23] F. D. M. Haldane, Phys. Rev. Lett. 51, 605 (1983).

[24] T. T. Wu and C. N. Yang, Nucl. Phys. B 107, 365 (1976).

[25] I. Peschel, J. Phys. A 36, L205 (2003).

[26] Note that due to our choice of equal-sized regions $A$ and $B$, degeneracies occur in the RSES of the IQH states. These degeneracies are associated with particle-hole symmetry in the occupations of the single-particle orbitals in Eq. (1). Such particle-hole degeneracy is no longer present once interactions are introduced [see, e.g., Fig. 1(b)].

[27] I. D. Rodríguez, S. C. Davenport, S. H. Simon, and J. K. Slingerland, Phys. Rev. B 88, 155307 (2013).

[28] G. J. Sreejith, S. Jolad, D. Sen, and J. K. Jain, Phys. Rev. B 84, 245104 (2011). 